\title{
无过渡金属参与的三芳基膦化合物合成新方法研究
}

\author{
尹清 于晓强* 包 明 \\ (大连理工大学精细化工国家重点实验室 大连 116024)
}

\begin{abstract}
摘要 三芳基膦化合物在医药合成、过渡金属催化等方面扮演十分重要的角色. 报道了碱促进的芳基卤代物与烷基取 代的二芳基膦的 $\mathrm{C}\left(\mathrm{sp}^{2}\right)-\mathrm{P}$ 键偶联新方法合成三芳基膦. 该方法具有无过渡金属催化剂参与、反应底物来源广泛、后处 理简洁等优点.
\end{abstract}

关键词 无过渡金属; 烷基取代的二芳基膦; 芳基卤代物; 三芳基膦化合物

\section{A Novel Method for the Synthesis Triarylphosphines under Transition-Metal-Free Conditions}

\author{
Yin, Qing Yu, Xiaoqiang* Bao, Ming \\ ( State Key Laboratory of Fine Chemicals, Dalian University of Technology, Dalian 116024)
}

\begin{abstract}
Triarylphosphines play an important role in pharmaceutical synthesis and transition-meal-catalyzed reactions. In this paper, a novel method for the synthesis of triarylphosphines via base-promoted $\mathrm{C}\left(\mathrm{sp}^{2}\right)-\mathrm{P}$ cross-coupling reactions of alkyldiphenylphosphines with aryl halides is described. The transition-metal-free reaction condition, readily available starting materials and experimental simplicity are the features of the novel method.

Keywords transition metal-free; alkyldiphenylphosphines; aryl halides; triarylphosphines
\end{abstract}

三芳基膦类化合物是一类非常重要的配体，被广泛 地应用于过渡金属催化的偶联反应中 ${ }^{[1]}$. 另外，三芳基 膦化合物还可以作为催化剂, 催化多种类型的有机合成 反应 ${ }^{[2]}$. 因此, 开发三芳基膦类化合物合成新方法一直 是有机合成化学领域中的研究热点之一.

传统合成三芳基膦类化合物的方法包括: 格氏试剂 法/锂试剂法 ${ }^{[3]} 、$ Friedel-Crafts 法 $^{[4]}$ 和三芳基膦氧化物还 原法 ${ }^{[5]}$. 虽然上述方法合成工艺已成熟, 但还存在反应 条件苛刻、官能团兼容性不好、反应选择性差等缺点尚 未得到有效解决. 近年来, 过渡金属催化 $\mathrm{C}-\mathrm{P}$ 键偶联 反应合成三芳基膦化合物得到了长足的发展, 然而, 具 有毒性、价格昂贵的金属催化剂(如钯 ${ }^{[6]}$ 、镍 ${ }^{[7]}$ 、铜 ${ }^{[8]}$ 等) 的使用, 是实现这种转化的关键; 另外, 二苯基 膦 ${ }^{[8 \mathrm{a}, 8 \mathrm{~b}]}$ (空气中可自燃) 或有机锡膦化合物 ${ }^{[6 \mathrm{e}, 9]}$ 的使用必 不可少. 2013 年, Yang 课题组 ${ }^{[10]}$ 报道了镍催化有机硅膦 化合物与碘代芳烃的 $\mathrm{C}-\mathrm{P}$ 键偶联反应合成三芳基膦. 2016 年, Wang 课题组 ${ }^{[11]}$ 实现了钯催化酰基膦化合物与
碘代芳烃的 $\mathrm{C}-\mathrm{P}$ 键偶联反应合成三芳基膦，由于有机 硅膦化合物和酰基膦化合物稳定性较好，因此上述两种 方法均可稳定高效率地得到目标产物. 但有机硅膦化合 物和酰基膦化合物都需要提前制备且合成步骤复杂. 另 外, Jackson 课题组 ${ }^{[12]}$ 实现了硅胶负载碱金属促进三芳 基膦与卤代物反应，制备二芳基膦化合物. 本文报道了 新型的碱促进芳基卤代物与烷基取代的二芳基膦的 $\mathrm{C}\left(\mathrm{sp}^{2}\right)$ - $\mathrm{P}$ 键偶联反应合成三芳基膦(图 1). 该方法具有 无过渡金属催化剂参与, 反应底物来源广泛，后处理简 洁等优点，为三芳基膦类化合物的合成提供了一条新的 思路.

\section{1 结果与讨论}

\section{1 反应条件的优化}

以 1,2-双(二苯基膦)乙烷 $(\mathbf{1 a}, 0.3 \mathrm{mmol}$ )与碘代苯 $(2 \mathrm{a}, 0.6 \mathrm{mmol})$ 为底物的反应为模型反应，考察了不同类 型碱、溶剂等对 $\mathrm{C}-\mathrm{P}$ 键偶联反应的影响, 实验结果如

\footnotetext{
* Corresponding author. E-mail: yuxiaoqiang@dlut.edu.cn

Received April 2, 2019; revised April 26, 2019; published online May 21, 2019.

Project supported by the National Natural Science Foundation of China (Nos. 21572028, 21573032).

国家自然科学基金(Nos. 21572028, 21573032)资助项目
} 


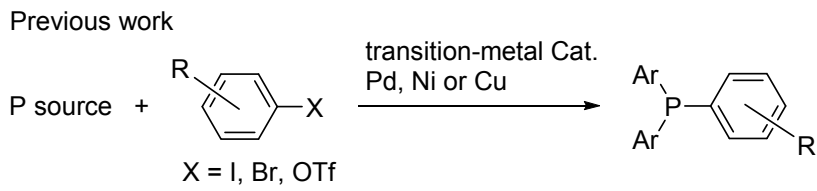

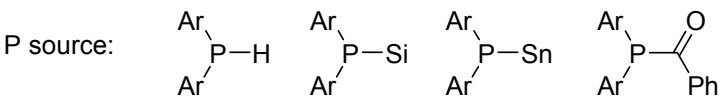

This work

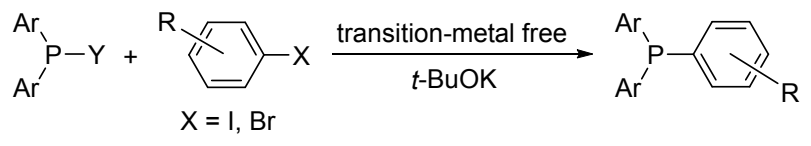

$\mathrm{Y}=\mathrm{CH}_{2} \mathrm{CH}_{3}, \mathrm{CH}_{2} \mathrm{CH}_{2} \mathrm{PPh}_{2}, \mathrm{CH}_{2} \mathrm{CH}_{2} \mathrm{CH}_{2} \mathrm{PPh}_{2}, \mathrm{CH}_{2} \mathrm{CH}_{2} \mathrm{CH}_{2} \mathrm{CH}_{2} \mathrm{PPh}_{2}$

图 1 三芳基膦化合物的合成方法

Figure 1 Pathways for synthesis of tertiarylphosphines

表 1 所示. 以二甲基亚砜(DMSO)为溶剂、 $\mathrm{Cs}_{2} \mathrm{CO}_{3}$ 为碱, 在 $\mathrm{N}_{2}$ 氛围下 $115{ }^{\circ} \mathrm{C}$ 反应 $6 \mathrm{~h}$, 得到目标化合物三苯基膦 的分离收率为 $39 \%$ (Entry 1). 其它无机碱 $\mathrm{K}_{2} \mathrm{CO}_{3}$ 、 $\mathrm{KOAc} 、 \mathrm{~K}_{3} \mathrm{PO}_{4} 、{ }^{t} \mathrm{BuONa}$ 和 ${ }^{t} \mathrm{BuOK}$, 也被分别应用于该 反应中, 发现当使用 ${ }^{t} \mathrm{BuOK}$ 时, 反应收率最高, 可达到 $70 \%$ (Entry 6). 另外, 有机碱吡啶、三乙胺、四甲基乙二 胺(TMEDA)和 2,2-联吡啶(2,2'-bpy)也先后被应用于该 反应中. 结果显示, 在有机碱的存在下反应也可以发生, 其目标产物的收率为 $23 \% \sim 42 \%$. 由此确定 ${ }^{t} \mathrm{BuOK}$ 为反 应最佳的碱.

\section{表 1 反应条件考察 ${ }^{a}$}

Table 1 Reaction condition screening

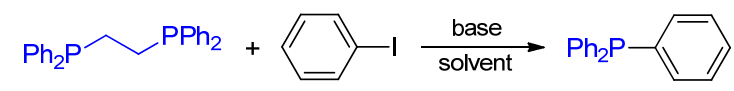

$1 a$

2a 3a

\begin{tabular}{|c|c|c|c|}
\hline Entry & Base & Solvent & Yield $^{b} / \%$ \\
\hline 1 & $\mathrm{Cs}_{2} \mathrm{CO}_{3}$ & DMSO & 39 \\
\hline 2 & $\mathrm{~K}_{2} \mathrm{CO}_{3}$ & DMSO & $\mathrm{NR}^{c}$ \\
\hline 3 & KOAc & DMSO & $\mathrm{NR}^{c}$ \\
\hline 4 & $\mathrm{~K}_{3} \mathrm{PO}_{4}$ & DMSO & 45 \\
\hline 5 & ${ }^{t} \mathrm{BuONa}$ & DMSO & 50 \\
\hline 6 & ${ }^{t} \mathrm{BuOK}$ & DMSO & 70 \\
\hline 7 & Pyridine & DMSO & 23 \\
\hline 8 & $\mathrm{Et}_{3} \mathrm{~N}$ & DMSO & 42 \\
\hline 9 & TMEDA & DMSO & 29 \\
\hline 10 & 2,2'-bpy & DMSO & 30 \\
\hline 11 & ${ }^{t} \mathrm{BuOK}$ & DMF & Trace \\
\hline 12 & ${ }^{t} \mathrm{BuOK}$ & DMAc & 33 \\
\hline 13 & ${ }^{t} \mathrm{BuOK}$ & NMP & Trace \\
\hline 14 & ${ }^{t} \mathrm{BuOK}$ & HMPA & $\mathrm{NR}^{c}$ \\
\hline 15 & ${ }^{t} \mathrm{BuOK}$ & Tolune & $\mathrm{NR}^{c}$ \\
\hline 16 & ${ }^{t} \mathrm{BuOK}$ & 1,4-Dioxane & $\mathrm{NR}^{c}$ \\
\hline
\end{tabular}

${ }^{a}$ Unless otherwise noted, all the reactions were conducted in solvent $(3.0 \mathrm{~mL})$ with 1a $(0.3 \mathrm{mmol})$ and $2 \mathbf{a}(0.6 \mathrm{mmol})$, additive $(1.05 \mathrm{mmol})$, in $\mathrm{N}_{2}$ atmosphere at $115{ }^{\circ} \mathrm{C}$ for $6 \mathrm{~h} .{ }^{b}$ Isolated yield. ${ }^{c}$ No reaction.
随后分别考察了 $N, N$-二甲基甲酰胺(DMF), N,N-二 甲基乙酰胺(DMAc)， $N$-甲基吡咯烷酮(NMP)，六甲基磷 酰三胺(HMPA)，甲苯(toluene)和 1,4-二氧六环(1,4-dioxane)对反应的影响. 发现上述溶剂的反应效果均不及 DMSO (Entries 11 16 vs Entry 6). 由此确定 DMSO 为 最佳的反应溶剂.

在确定了反应溶剂和碱之后，分别考察了碱的用 量、反应时间和反应温度对反应收率的影响. 发现通过 增加碱的用量、延长反应时间或提高反应温度等手段并 不能提高反应收率. 最后, 确定了最优反应条件是: 1,2双 (二苯基膦)乙烷 (1a，0.3 mmol)，碘代苯 (2a，0.6 $\mathrm{mmol}),{ }^{t} \mathrm{BuOK}(1.05 \mathrm{mmol})$ 为碱, $\mathrm{DMSO}$ 为溶剂, 在氮气 氛围下 $115{ }^{\circ} \mathrm{C}$ 反应 $6 \mathrm{~h}$.

\section{2 底物的拓展}

在最佳反应条件下，考察了不同取代基团的卤代芳 烃的反应活性, 结果见表 2 所示. 碘苯的芳环上带有甲 基或苯基均能很好地适用该方法(Entries $2 \sim 6$ ), 而碘苯 的芳环上含有拉电子取代基如硝基和氰基等的底物则 不适用于该反应(Entries 7，8). 芳环上取代基的电子效 应对该反应有着至关重要的影响，而空间位阻效应对反 应结果没有影响(Entries 3 5 vs Entry 2). 碘代萗同样 适用于该反应, 生成目标化合物 $3 \mathrm{~g}$ 的收率为 $53 \%$. 另 外，尝试了用溴代芳烃替代碘代芳烃来进行 $\mathrm{C}-\mathrm{P}$ 键偶 联反应. 同样, 溴苯的芳环上带有甲基或苯基均能顺利 地进行 $\mathrm{C}-\mathrm{P}$ 键偶联(Entries 11 15). 当底物为芳环上 含有羰基的溴代芳烃(2p)时，则不能得到相应的三芳基 膦化合物(Entry 16). 溴代菜同样适用于该反应(Entry 17).

在最佳反应条件下，还考察了不同结构的膦源对反 应的影响, 结果如表 3 所示. 当使用 1,3-双(二苯基膦) 丙烷 $(1 b)$ 代替 $1 a$ ，与碘代芳烃 $2 a 、 2 b$ 和 $2 c$ 分别进行碱 促进的 $\mathrm{C}-\mathrm{P}$ 键偶联反应时, 反应可以顺利地发生，得 到相应的三芳基膦化合物 3a、3b 和 3c，收率为 40\% 53\% (Entries 1 3). 其它类型的膦源, 如 1,4-双 (二苯基 膦)丁烷 (1c) 和乙基二苯膦 (1d) 同样适用于该反应 (Entries 4 9). 然而, 当使用甲基二苯膦(1e)作为膦源 时, $\mathrm{C}-\mathrm{P}$ 键偶联则不能发生(Entries 10 12).

\section{3 反应机理研究}

根据文献报道和本反应的实验现象，推测该反应的 机理为: 首先碘苯在强碱 ${ }^{t} \mathrm{BuOK}$ 以及高温的作用下, 形 成碘苯自由基负离子中间体 $\mathbf{A}^{[13]}$, 进一步形成苯基自由 基 $\mathbf{B}$, 自由基 $\mathbf{B}$ 对 1,2-双(二苯基膦)乙烷进行自由基加 成, 生成膦自由基中间体 $\mathbf{C}$, 接下来 $\mathrm{C}-\mathrm{P}$ 键均裂生成 目标化合物三芳基膦和自由基中间体 $\mathbf{D}$, 中间体 $\mathbf{D}$ 解离 
表 2 以 1,2-双 (二苯基膦) 乙烷为膦源的三芳基膦衍生物合成 ${ }^{a}$

Table 2 Synthesis of arylphosphine compound with 1,2-bis(diphenylphosphino)ethane<smiles>[R]c1ccc(Pc2ccccc2)cc1</smiles>

$1 a$

2

$\mathbf{3 a} \sim \mathbf{3 h}$

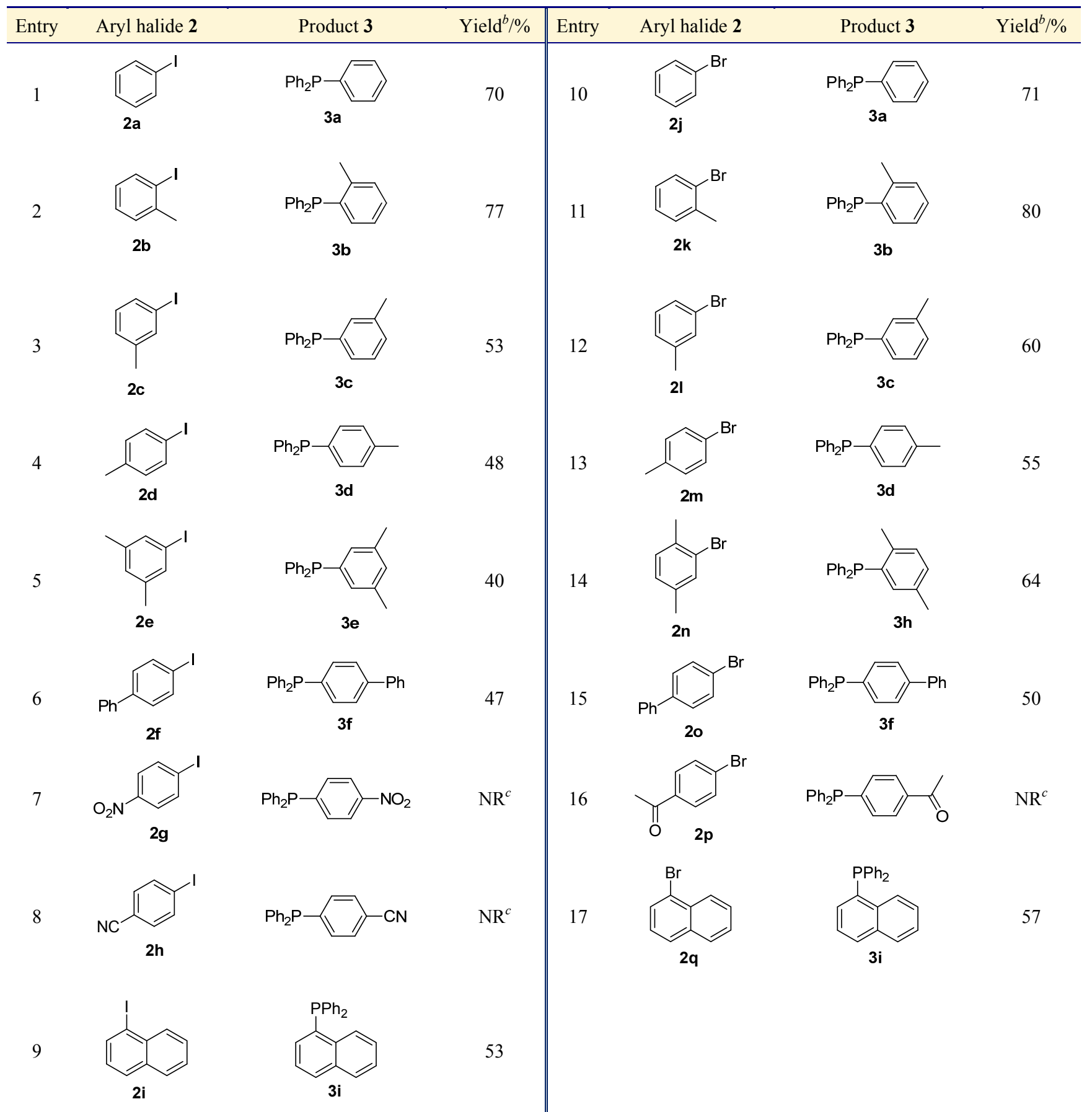

$\overline{{ }^{a}}$ Unless otherwise noted, all the reactions were conducted in DMSO $(3.0 \mathrm{~mL})$ with 1a $(0.3 \mathrm{mmol}), \mathbf{2}(0.6 \mathrm{mmol})$ and ${ }^{t} \mathrm{BuOK}(1.05 \mathrm{mmol})$ in $\mathrm{N}_{2}$ atmosphere at $115{ }^{\circ} \mathrm{C}$ for $6 \mathrm{~h} .{ }^{b}$ Isolated yield. ${ }^{c}$ No reaction.

出一个氢自由基形成乙烯基二苯基膦，最后氢自由基与 叔丁氧自由基结合成叔丁醇. 苯基自由基 $\mathbf{B}$ 可由 Tempo 捕获 ${ }^{[14]}$, 副产物乙烯基二苯基膦和叔丁醇均可被 GC-MS 检测到(相关谱图见辅助材料).

\section{2 结论}

开发了在无过渡金属催化剂存在的条件下，芳基卤 代物与烷基取代二芳基膦化合物的 $\mathrm{C}\left(\mathrm{sp}^{2}\right)-\mathrm{P}$ 键偶联反 应来构建三芳基膦. 反应的最佳条件为: 在氮气保护下, 
表 3 其他烷基取代二芳基膦为膦源的芳基膦衍生物的合成 ${ }^{a}$

Table 3 Synthesis of arylphosphine compound with other alkyldiphenylphosphines

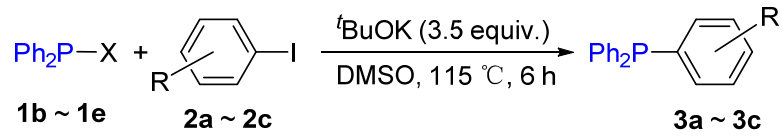

\begin{tabular}{|c|c|c|c|c|c|c|c|c|c|}
\hline Entry & Phosphorus source 1 & Aryl halide 2 & Product 3 & Yield $^{b} / \%$ & Entry & Phosphorus source 1 & Aryl halide 2 & Product 3 & Yield $^{b} / \%$ \\
\hline 1 & & $2 \mathbf{a}$ & $3 \mathbf{a}$ & 46 & 7 & & $2 a$ & $3 \mathbf{a}$ & 44 \\
\hline 2 & & $2 b$ & $3 \mathbf{b}$ & 53 & 8 & $\mathrm{Ph}^{-}{ }^{-{ }_{-}} \mathrm{Ph}$ & $2 \mathbf{b}$ & $3 \mathbf{b}$ & 47 \\
\hline 3 & $1 b$ & $2 \mathrm{c}$ & $3 \mathrm{c}$ & 40 & 9 & 1d & $2 \mathrm{c}$ & $3 \mathbf{c}$ & 40 \\
\hline 4 & $\mathrm{PPh}_{2}$ & $2 a$ & $3 \mathbf{a}$ & 36 & 10 & & $2 a$ & - & $\mathrm{NR}^{c}$ \\
\hline 5 & & $2 \mathbf{b}$ & $3 \mathbf{b}$ & 40 & 11 & $\mathrm{Ph}^{-}{ }^{-} \mathrm{Ph}$ & $2 b$ & - & $\mathrm{NR}^{c}$ \\
\hline 6 & 1c & $2 \mathrm{c}$ & $3 c$ & 30 & 12 & & $2 c$ & - & $\mathrm{NR}^{c}$ \\
\hline
\end{tabular}

${ }^{a}$ Unless otherwise noted, all the reactions were conducted in DMSO $(3.0 \mathrm{~mL})$ with $\mathbf{1}(0.3 \mathrm{mmol}), 2(0.6 \mathrm{mmol})$ and ${ }^{t} \mathrm{BuOK}(1.05 \mathrm{mmol})$ in $\mathrm{N}_{2}$ atmosphere at $115{ }^{\circ} \mathrm{C}$ for 6 h. ${ }^{b}$ Isolated yield. ${ }^{c}$ No reaction.

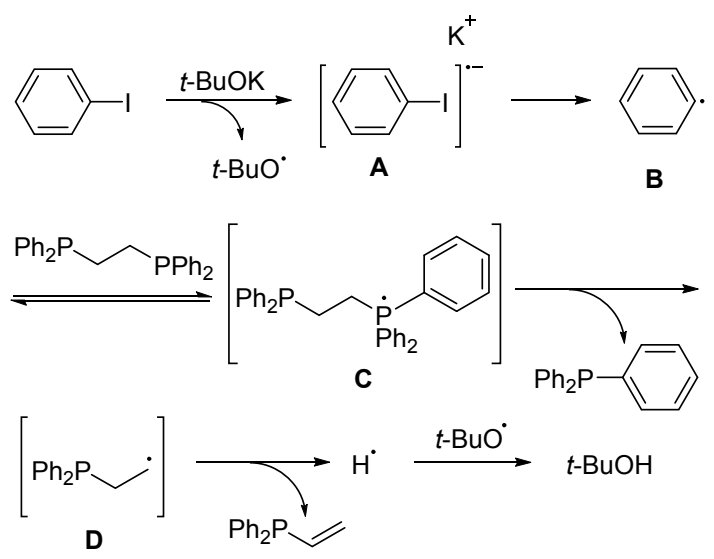

图 2 可能的机理推测

Figure 2 Plausible mechanism

$115{ }^{\circ} \mathrm{C}$, 烷基取代二芳基膦、芳基卤代物和 ${ }^{t} \mathrm{BuOK}$ 的物 质的量比例为 $1: 2: 3.5$, DMSO 溶液中反应 $6 \mathrm{~h}$. 这种 ${ }^{t} \mathrm{BuOK}$ 促进的 $\mathrm{C}-\mathrm{P}$ 键偶联反应, 为三芳基膦类化合物 的合成提供了一条新的思路.

\section{3 实验部分}

\section{1 仪器与试剂}

${ }^{1} \mathrm{H}$ NMR, ${ }^{13} \mathrm{C}$ NMR 和 ${ }^{31} \mathrm{P}$ NMR 谱均使用瑞士 Bruker 公司 AVANCE II-400 型超导核磁共振仪测定. ${ }^{1} \mathrm{H}$ NMR (400 MHz) 以四甲基硅烷(TMS)为内标, 溶剂为

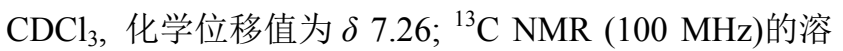
剂为 $\mathrm{CDCl}_{3}$, 化学位移值为 $\delta 77.16 ;{ }^{31} \mathrm{P} \mathrm{NMR}(162 \mathrm{MHz})$ 的溶剂为 $\mathrm{CDCl}_{3}, 85 \%$ 磷酸作为内标. GC-MS 谱图使用 美国 Agilent 公司气相色谱/三重串联四极杆质谱联用 仪 7000B 测试. 实验所用试剂购于伊诺凯化学和安耐 吉化学, 均为分析纯, 其中 DMSO、DMF、DMAc、NMP、 HMPA 均为隔膜瓶包装的无水超干溶剂, 甲苯、1,4-二
氧六环是实验室重蒸精制后的无水无氧溶剂.

\section{2 实验方法}

\subsection{1目标化合物 $\mathbf{3 a} \sim \mathbf{3 h}$ 的合成}

向 $25 \mathrm{~mL}$ Schlenk 反应器中加入 $1(0.3 \mathrm{mmol})$ 、叔丁 醇钾(1.05 mmol, $0.1178 \mathrm{~g})$, 在 $\mathrm{N}_{2}$ 下加入溶剂 DMSO (3 $\mathrm{mL})$ 以及 $2(0.6 \mathrm{mmol})$, 密封, 反应体系在无水无氧的条 件下 $115{ }^{\circ} \mathrm{C}$ 连续反应 $6 \mathrm{~h}$. 反应结束后, 冷却至室温, 反 应混合液用水和乙酸乙酯萃取三次，有机相用无水硫酸 钠干燥 $0.5 \mathrm{~h}$ 后, 减压蒸馏除去溶剂, 硅胶柱层析分离 (洗脱剂: 石油醚), 收集流出液并减压蒸馏除去溶剂, 真空干燥后得到白色固体 $\mathbf{3 a} \sim \mathbf{3 h}$.

三苯基膦(3a): 白色固体 $(55.0 \mathrm{mg})$, 底物为碘代苯 时产率为 70\%. m.p. $78 \sim 79{ }^{\circ} \mathrm{C}$ (lit. ${ }^{[6 \mathrm{~d}]}$ m.p. $79 \sim 80{ }^{\circ} \mathrm{C}$ ); ${ }^{1} \mathrm{H}$ NMR $\left(\mathrm{CDCl}_{3}, 400 \mathrm{MHz}\right) \delta: 7.34 \sim 7.28(\mathrm{~m}, 15 \mathrm{H}) ;{ }^{13} \mathrm{C}$ NMR $\left(\mathrm{CDCl}_{3}, 100 \mathrm{MHz}\right) \delta: 137.3,137.2,134.0,133.8$, 128.8, 128.7, 128.6; ${ }^{31} \mathrm{P} \mathrm{NMR}\left(\mathrm{CDCl}_{3}, 162 \mathrm{MHz}\right) \delta:-$ $5.44(\mathrm{~s})$.

2-甲苯基二苯基膦(3b)：白色固体(63.5 mg), 底物 为邻碘甲苯时产率为 $77 \%$. m.p. $65 \sim 67{ }^{\circ} \mathrm{C}$ (lit. ${ }^{[6 \mathrm{~d}]} \mathrm{m} . \mathrm{p}$. $\left.65 \sim 66{ }^{\circ} \mathrm{C}\right) ;{ }^{1} \mathrm{H} \mathrm{NMR}\left(\mathrm{CDCl}_{3}, 400 \mathrm{MHz}\right) \delta: 7.33 \sim 7.22$ $(\mathrm{m}, 12 \mathrm{H}), 7.09 \sim 7.05(\mathrm{~m}, 1 \mathrm{H}), 6.77(\mathrm{dd}, J=7.6,4.7 \mathrm{~Hz}$, 1H), 2.39 (s, 3H); ${ }^{13} \mathrm{C} \mathrm{NMR}\left(\mathrm{CDCl}_{3}, 100 \mathrm{MHz}\right) \delta: 142.4$, $142.2,136.4,136.3,134.2,134.0,132.8,130.2,130.1$, $128.9,128.8,128.7,128.6,126.1,21.2 ;{ }^{31} \mathrm{P} \mathrm{NMR}\left(\mathrm{CDCl}_{3}\right.$, $162 \mathrm{MHz}) \delta:-13.32(\mathrm{~s})$.

3-甲苯基二苯基膦(3c)：白色固体(43.7 mg), 底物 为间碘甲苯时产率为 $53 \%$. m.p. $48 \sim 50{ }^{\circ} \mathrm{C}$ (lit. ${ }^{[6 \mathrm{~d}]}$ m.p. $\left.50 \sim 51{ }^{\circ} \mathrm{C}\right) ;{ }^{1} \mathrm{H} \mathrm{NMR}\left(\mathrm{CDCl}_{3}, 400 \mathrm{MHz}\right) \delta: 7.34 \sim 7.28$ $(\mathrm{m}, 10 \mathrm{H}), 7.24 \sim 7.20(\mathrm{~m}, 1 \mathrm{H}), 7.18 \sim 7.14(\mathrm{~m}, 2 \mathrm{H})$, $7.09 \sim 7.05(\mathrm{~m}, 1 \mathrm{H}), 2.30(\mathrm{~s}, 3 \mathrm{H}) ;{ }^{13} \mathrm{C} \mathrm{NMR}\left(\mathrm{CDCl}_{3}, 100\right.$ 
MHz) $\delta: 138.1,137.4,134.7,134.5,134.0,133.8,132.2$, $131.0,130.8,129.7,128.8,128.6,128.5,21.6 ;{ }^{31} \mathrm{P}$ NMR $\left(\mathrm{CDCl}_{3}, 162 \mathrm{MHz}\right) \delta$ : $-5.39(\mathrm{~s})$.

4-甲苯基二苯基膦(3d): 白色固体(39.6 mg), 底物 为对碘甲苯时产率为 $48 \%$. m.p. $68 \sim 70{ }^{\circ} \mathrm{C}$ (lit. ${ }^{[6 \mathrm{~d}]}$ m.p. $\left.68 \sim 69{ }^{\circ} \mathrm{C}\right) ;{ }^{1} \mathrm{H}$ NMR $\left(\mathrm{CDCl}_{3}, 400 \mathrm{MHz}\right) \delta: 7.33 \sim 7.27$ $(\mathrm{m}, 10 \mathrm{H}), 7.24 \sim 7.20(\mathrm{~m}, 2 \mathrm{H}), 7.15(\mathrm{~d}, J=7.4 \mathrm{~Hz}, 2 \mathrm{H})$, $2.34(\mathrm{~s}, 3 \mathrm{H}) ;{ }^{13} \mathrm{C} \mathrm{NMR}\left(\mathrm{CDCl}_{3}, 100 \mathrm{MHz}\right) \delta: 139.0,137.7$, $137.6,134.1,134.0,133.8,133.6,129.5,129.4,128.7$, 128.6, 128.5, 21.5; ${ }^{31} \mathrm{P} \mathrm{NMR}\left(\mathrm{CDCl}_{3}, 162 \mathrm{MHz}\right) \delta$ : -6.34 (s).

3,5-二甲基苯基二苯基膦 (3e $)^{[6 \mathrm{~d}]}$ : 无色油状液体 (34.6 mg), 产率 $40 \% .{ }^{1} \mathrm{H} \mathrm{NMR}\left(\mathrm{CDCl}_{3}, 400 \mathrm{MHz}\right) \delta$ : $7.27 \sim 7.16(\mathrm{~m}, 10 \mathrm{H}), 6.90 \sim 6.85(\mathrm{~m}, 3 \mathrm{H}), 2.18(\mathrm{~s}, 6 \mathrm{H})$; ${ }^{13} \mathrm{C} \mathrm{NMR}\left(\mathrm{CDCl}_{3}, 100 \mathrm{MHz}\right) \delta: 138.1,138.0,137.7,137.6$, $133.9,133.8,131.7,131.6,130.8,128.7,128.6,128.5$, 21.4; ${ }^{31} \mathrm{P}$ NMR $\left(\mathrm{CDCl}_{3}, 162 \mathrm{MHz}\right) \delta$ : $-5.30(\mathrm{~s})$.

4-二苯基膦联苯(3f): 白色固体(47.7 mg), 底物为对 碘联苯时产率为 $47 \%$. m.p. $84 \sim 85{ }^{\circ} \mathrm{C}$ (lit. ${ }^{[6 \mathrm{~d}]}$ m.p. 83 $\left.84{ }^{\circ} \mathrm{C}\right) ;{ }^{1} \mathrm{H} \mathrm{NMR}\left(\mathrm{CDCl}_{3}, 400 \mathrm{MHz}\right) \delta: 7.61 \sim 7.57(\mathrm{~m}, 4$ $\mathrm{H}), 7.48 \sim 7.43(\mathrm{~m}, 3 \mathrm{H}), 7.40 \sim 7.34(\mathrm{~m}, 12 \mathrm{H}) ;{ }^{13} \mathrm{C} \mathrm{NMR}$ $\left(\mathrm{CDCl}_{3}, 100 \mathrm{MHz}\right) \delta: 141.6,140.7,137.4,137.3,136.3$, $136.2,134.4,134.2,134.0,133.8,129.0,128.7,128.6$, $127.7,127.3,127.2 ;{ }^{31} \mathrm{P}$ NMR $\left(\mathrm{CDCl}_{3}, 162 \mathrm{MHz}\right) \delta$ : $6.01(\mathrm{~s})$.

1-菜基二苯基膦(3g)：白色固体(49.6 mg), 底物为 1-碘代萗时产率为 53\%. m.p. $121 \sim 123{ }^{\circ} \mathrm{C}$ (lit. ${ }^{[6 \mathrm{~d}]} \mathrm{m} . \mathrm{p}$. $\left.122 \sim 123{ }^{\circ} \mathrm{C}\right) ;{ }^{1} \mathrm{H}$ NMR $\left(\mathrm{CDCl}_{3}, 400 \mathrm{MHz}\right) \delta: 8.40$ (dd, $J=8.4,4.3 \mathrm{~Hz}, 1 \mathrm{H}), 7.87 \sim 7.83(\mathrm{~m}, 2 \mathrm{H}), 7.50 \sim 7.41(\mathrm{~m}$, $2 \mathrm{H}), 7.37 \sim 7.24(\mathrm{~m}, 11 \mathrm{H}), 7.00(\mathrm{dd}, J=7.0,5.1 \mathrm{~Hz}, 1 \mathrm{H})$; ${ }^{13} \mathrm{C} \mathrm{NMR}\left(\mathrm{CDCl}_{3}, 100 \mathrm{MHz}\right) \delta: 136.5,136.4,135.5,135.3$, $134.4,134.2$, 133.6, 133.5, 132.2, 129.6, 129.0, 128.7, 128.6, 126.4, 126.1, 125.7; ${ }^{31} \mathrm{P} \mathrm{NMR}\left(\mathrm{CDCl}_{3}, 162 \mathrm{MHz}\right) \delta$ : $-14.26(\mathrm{~s})$.

2,5-二甲基苯基二苯基膦(3h)：白色固体(55.3 mg), 产率 64\%. m.p. 81 $82{ }^{\circ} \mathrm{C}$ (lit. ${ }^{[14]}$ m.p. $81 \sim 83{ }^{\circ} \mathrm{C}$ ); ${ }^{1} \mathrm{H}$ NMR $\left(\mathrm{CDCl}_{3}, 400 \mathrm{MHz}\right) \delta: 7.34 \sim 7.24(\mathrm{~m}, 10 \mathrm{H}), 7.09 \sim$ 7.04 (m, $2 \mathrm{H}), 6.57$ (dd, $J=4.9,1.7 \mathrm{~Hz}, 1 \mathrm{H}), 2.34$ (s, $3 \mathrm{H})$, 2.15 (s, $3 \mathrm{H}) ;{ }^{13} \mathrm{C} \mathrm{NMR}\left(\mathrm{CDCl}_{3}, 100 \mathrm{MHz}\right) \delta: 139.3,139.1$, $136.6,136.5,135.4,134.2,134.0,133.4,130.2$, 130.1, 129.6, 128.8, 128.7, 128.6, 21.2, 21.1; ${ }^{31} \mathrm{P} \mathrm{NMR}\left(\mathrm{CDCl}_{3}\right.$, $162 \mathrm{MHz}) \delta$ : $-13.09(\mathrm{~s})$.

辅助材料(Supporting Information) 三芳基膦化合物 的 ${ }^{1} \mathrm{H} \mathrm{NMR},{ }^{13} \mathrm{C} \mathrm{NMR},{ }^{31} \mathrm{P} \mathrm{NMR}$ 的谱图, 证明机理的
Tempo 捕捉自由基的 ${ }^{1} \mathrm{H} \mathrm{NMR},{ }^{13} \mathrm{C} \mathrm{NMR}$ 的谱图和反应 液混合物的 GC-MS 谱图. 这些材料可以免费从本刊网 站(http://sioc-journal.cn/)上下载.

\section{References}

[1] (a) Martin, R.; Buchwald, S. L. Acc. Chem. Res. 2008, 41, 1461. (b) Beletskaya, I. P.; Kazankova, M. A. Russ. J. Org. Chem. 2002, $38,1391$.

(c) Kosolapoff, G. M.; Maier, L. In Organic Phosphorus Compounds, Vol. 1, 2nd ed., Wiley-Interscience, New York, 1972, p. 545 .

(d) Ojima, I.; Clos, N.; Bastos, C. Tetrahedron 1989, 45, 6901. (e) Surry, D. S.; Buchwald, S. L. Angew. Chem., Int. Ed. 2008, 47, 6338 .

[2] (a) Tran, U. P. N.; Hock, K, J.; Gordon, C. P. Chem. Commun. 2017, 53,4950 .

(b) Zheng, F.; Leung, T.; Chan, K. Chem. Commun. 2016, 52, 10767. (c) Shih, H. W.; Prescher, J. A. J. Am. Chem. Soc. 2015, 137, 10036 .

[3] (a) Jiménez, M. V.; Pérez-Torrente, J. J. Synthesis 2009, 1916. (b) Zakharkin, L. I.; Anikina, E. V. Izv. Akad. Nauk SSSR, Ser. Khim. 1990, 3, 714.

(c) Unruh, J. D.; Christenson, J. R. J. Mol. Catal. 1982, 14, 19. (d) Imamoto, T.; Kikuchi, S. I.; Miura, T.; Wada, Y. Org. Lett. 2001, 3,87 .

[4] (a) Michaelis, A. Chem. Ber. 1879, 12, 1009.

(b) Kosolapoff, G. M.; Huber, W. F. J. Am. Chem. Soc. 1947, 69, 2020.

(c) Gefter, E. L. Zh. Obsh. Khim. 1958, 28, 1338

[5] (a) Li, Y.; Das, S.; Beller, M. J. Am. Chem. Soc. 2012, 134, 9727. (b) Buonomo, J. A.; Eiden, C. G.; Aldrich, C. C. Chem.-Eur. J. 2017, 23, 14434.

(c) Herault, D.; Nguyen, D. H.; Nuel, D. Chem. Soc. Rev. 2015, 44, 2508.

(d) Rinehart, N. I.; Kendall, A. J.; Tyler, D. R. Organometallics 2018, 37, 182.

(e) Tunney, S. E.; Stille, J. K. J. Org. Chem. 1987, 52, 748.

[6] (a) Herd, O.; Stelzer, O. J. Organomet. Chem. 1996, 522, 69. (b) Machnitzki, P.; Stelzer, O. Eur. J. Inorg. Chem. 1998, 7, 1029. (c) Nowrouzi, N.; Keshtgar, S.; Jahromi, E. B. Tetrahedron Lett. 2016, 57,348 .

(d) Xu, Z.; Wang, P.; Cai, M. J. Org. Chem. 2018, 866, 50

(e) Stadler, A.; Kappe, C. O. Org. Lett. 2002, 4, 3541.

(f) Lian, Z.; Bhawal, B. N.; Yu, P.; Morandi, B. Science 2017, 356 , 1059.

[7] (a) Yang, J.; Chen, T.; Han, L. B. J. Am. Chem. Soc. 2015, 137, 1782 .

(b) Cai, D.; Payack, J. F.; Bender, D. R.; Hughes, D. L. J. Org. Chem. 1994, 59, 7180.

(c) Cai, D.; Payack, J. F.; Bender, D. R. Org. Synth. 1998, 76, 6. (d) Sun, M.; Zang, Y. S.; Hou, L. K. Eur. J. Org. Chem. 2014, 30, 6796.

[8] (a) Fang, Z.; Cai, M.; Lin, Y.; Zhao, H. Appl. Organomet. Chem. 2018, 32, 4417.

(b) Allen, D. V.; Venkataraman, D. J. Org. Chem. 2003, 68, 4590

(c) Gelman, D.; Jiang, L.; Buchwald, S. L. Org. Lett. 2003, 5, 2315.

(d) Rao, H. H.; Jin, Y.; Zhao, Y. F. Chem.- Eur. J. 2006, 12, 3636.

(e) Huang, C.; Tang, X.; Jiang, Y. Y.; Zhao, Y. F. J. Org. Chem. 2006, 71,5020 .

[9] (a) Li, Y.; Chakrabarty, S.; Studer, A. Angew. Chem., Int. Ed. 2016 55,802 .

(b) Schumann, H.; Jutzi, P.; Schmidt, M. Angew. Chem., Int. Ed. Engl. 1965, 4, 869 .

(c) Schumann, H.; Jutzi, P.; Schmidt, M. Angew. Chem. 1965, 77, 912.

[10] Li, Y. M.; Yang, S. D. Synthesis 2013, 1739. 
[11] (a) Yu, R. R.; Chen, X. Y.; Martin, S. F.; Wang, Z. Q. Org. Lett. 2017, 19, 1808.

(b) Yu, R. R.; Chen, X. Y.; Wang, Z. Q. Tetranedron Lett. 2016, 57, 3404.

(c) Yang, J. F.; Wu, H. Y.; Wang, Z. Q.; Saudi, J. Chem. Soc. 2016, 7,1319 .
[12] Nandi, P.; Dye, J. L.; Bentley, P.; Jackson, J. E. Org. Lett. 2009, 11, 1689.

[13] (a) Shirakawa, E.; Zhang, X.; Hayashi, T. Angew. Chem. Int. Ed. 2011, 50, 4671.

(b) Bunnett, J. F. Acc. Chem. Res. 1978, 11, 413.

[14] Schindlbauer, H. Monatsh. Chem. 1965, 96. 2058.

(Li, L.; Fan, Y.) 